\title{
The potential of cooperative communications to speed up disaster relief operations
}

\author{
Farouk Mezghani, Nathalie Mitton \\ Inria Lille \\ \{farouk.mezghani, nathalie.mitton\}@inria.fr
}

\begin{abstract}
This paper proposes FLY-COPE, a complete selforganization architecture that relies on cooperative communications and drone-assisted data collection, allowing a fast location of victims and rescuing operation organization in disaster relief operation. FLY-COPE mainly combines two components: i) a ground component that spontaneously emerges from any communicating devices (piece of infrastructure, mobile phone, etc) that cooperate to alert rescuers and remain all alive as long as possible and ii) an aerial component comprising UAV to communicate efficiently with ground devices. We show by simulation and/or by experimentation that each component of FLY-COPE allows substantial energy saving for efficient and fast disaster response.

Index Terms-Disaster recovery, mobile devices, multi-tier cooperative communication, UAV, drone-based data relaying, energy-efficient communication
\end{abstract}

\section{INTRODUCTION}

When a natural or human disaster occurs, the first 72 hours, the golden relief time, are particularly critical to locate and rescue people [1]. To do so, communication from the victims to the rescuers and between rescuer is paramount. But in most cases, in such situations, communication networks (e.g., cellular base stations) are completely destroyed, damaged or saturated.

Recently, disseminating data in post-disaster situations has received increasing attention [2]-[6]. But we believe that none of these works exploits the full potential of autonomous wireless devices neither considers the full picture of the situation. For instance, non of these works leverages the availability of multiple communication technologies or provide bilateral communication. Indeed, most of solutions propose a kind of convergecast algorithm where data comes from the victims to the rescuer while the former may need to be comforted and kept aware of rescuers progression thanks to a backward communication (from the rescuer to the victims).

On the other hand, drones or UAV are more and more deployed in post-disaster situations [7]-[9], even if only to assess the damages during fires (see the Paris Notre Dame fire in April 2019), floods (see in North Carolina in 2018), earthquakes (see Philippines after Typhoon Haiyan in 2013), Hurricanes (see Haiti in Sandy in 2012), etc. During current drone interventions, drones are remotely controlled by humans. And, the research work investigating the use of drones for disaster reliefs [10], [11] solely address placement and optimization of UAVs without combining it with a dynamic data collection.
In this paper, we propose FLY-COPE, a complete alternative paradigm for situations where traditional infrastructure is not available. FLY-COPE allows survivors to reveal themselves for a duration as long as possible in a seamless way and rescuers to intercept their SOS and answer them. This global picture relies on cooperative communications between any still active communication device (personal and wearable devices owned by survivors, pieces of infrastructure such as Wifi access points, BTS, still active but disconnected from the core network, etc) and with unmanned aerial vehicles. It comprises a multi-tiers self-organization protocol that leverages the multiple communication technologies available on ground devices such as mobile phones and a UAV-assisted data collection. To the best of our knowledge, it is the first attempt to combine all individual components currently investigated in silo. Our global architecture enables bi-directional communications and allows the individual devices to save their energy as much as possible. It is based on our previous work [12], [13]. This paper presents this systemic architecture together with the evaluation of its components and derives some related open challenges.

The remainder of this paper is as follows. Section II briefly describes the state of the art of wireless communications used for disaster reliefs. An overview of our architecture FLYCOPE is presented in Section IV while both main components are detailed respectively in Sections V and VI. We present a set of open challenges and issues in Section VII. Finally, Section VIII concludes this paper.

\section{RELATED WORKS}

FLY-COPE is a global architecture to allow spontaneous communication between victims and rescuers. It combines different approaches and aims to optimize the global energy efficiency in order to allow individual devices to be active as long as possible. FLY-COPE comprises two components. To the best of our knowledge, it is the first attempt to combine the advantages of aerial and ground organization in communication for post-disaster response. We thus provide a brief overview of the literature work in each component.

\section{A. Ground component}

Most of the work in wireless sensor networks with mobile elements and ad-hoc networks aim at providing wireless communication during natural disaster phenomena [5], [14], [15]. But none of these works leverages the availability of multiple 
communication technologies and mainly assume homogeneous devices.

Moreover, the ubiquitous nature of smart devices such as smartphones is largely exploited by many works, whose main focus consists of extending the wireless connectivity coverage in areas with missing or damaged infrastructure [3], [16], considering a complementary approach than FLY-COPE.

Smart devices constitute one key element in survivor-rescuer systems [1], [17] - they send out location data of the survivors to rescue teams, for instance, as considered in FLY-COPE. Accordingly, the works in [18], [19] show how such entities communicate with each other via aerial base stations, i.e., UAVs that fly over a disaster area with on-board femtocells. However, such works provide no considerations on the energy-efficiency of the proposed solutions and consider selfish approaches in which each device directly communicate with the drone, without any ground self-organization.

\section{B. Aerial component}

From the aerial component perspective, existing work mainly focus on the deployment of a connected wireless UAVdrones [20].

Some works with flying ad-hoc networks present performance tradeoffs as a function of parameters such as the UAV height and placement to maximize the coverage in a multiUAV system [21], [22]. By contrast, we focus on a singleUAV system which requires no synchronization, placement map over a given area, or task scheduling among UAVs.

\section{SYSTEM MODEL}

\section{A. FLY-COPE ground component}

Our system considers a set of communicating devices. These latter could be survivors' personal devices or remaining infrastructure components. We assume that each device is equipped with a set of $\mathcal{N}=\left\{n_{u}, 1 \leq u \leq U\right\}$ communication technologies, with $U$ the number of available interfaces. These technologies can be ranked based on their range $r$ and energy consumption $c$ such that :

$$
c_{n_{1}}<c_{n_{u}}<c_{n_{U}} \text { and } r_{n_{1}}<r_{n_{u}}<r_{n_{U}}
$$

As an example, we can consider an off-the-shelf cell phone that is traditionally equipped with $U=4$ communication technologies: NFC, Bluetooth, Wifi and cellular (2G/3G/4G). These technologies can be ranked as NFC $\left(n_{1}\right)<$ Bluetooth $\left(n_{2}\right)<$ Wifi $\left(n_{3}\right)<$ cellular $\left(n_{4}\right)$ according to their respective ranges and energy consumption. We later use the terms of layer or tiers of level $l$ to refer to the $l^{\text {th }}$ rank.

\section{B. FLY-COPE aerial component}

Two kinds of drones can be used: fixed wings drones and multi-rotors drones as depicted on Figure 2. Currently, disaster relief operations call on multi-rotor drones since they allow stationary flight better manoeuvrability and adaptable speed. On the other hand, fixed wing drones are indeed less flexible and manoeuvrable but they can reach higher speed. We propose a two-step drone based data collection, each step calling for a kind of drone (See Section VI). The first step aims to locate in a fast and accurate way all potential survivors. The fixed-wing drones are used. The second step aims to maintain (intermittent) connectivity with survivors. The multirotor drones are used. We assume all drones are equipped with the $n_{U}$ (the upmost layer communication technology). In the case of mobile phones, this corresponds to a cellular connectivity $(2 \mathrm{G} / 3 \mathrm{G} / 4 \mathrm{G})$. This can be realized by mounting femto-cells on drones [10], [23].

\section{FLY-COPE ARCHITECTURE OVERVIEW}

Figure 1 illustrates our FLY-COPE architecture. It is composed of two main components: a ground component and an aerial component.

The ground component comprises the set of mobile devices owned by the survivors but also of all operational communication device (e.g. a base station (wifi access point, node $\mathrm{B}$, etc) which is still operational but which has been disconnected from the core network). These devices selforganize to share the communication energy costs among all of them. They activate in turn the most energy-greedy communication interfaces according to their individual energy reserves. For each communication interface, they identify cliques and only the representative of the clique activates its upper layer communication interface. This self-organization is more detailed in Section V.

At the upmost layer ( $n_{U}$-tiers), only a subset of survivors' devices are active. They can directly communicate with the drones which are equipped with the same technology. Since most of communication infrastructure components may be damaged, we can not guarantee that these upmost layer devices could reach a rescuer. Therefore, we use drones for this last step data collection. The drones compose the aerial component. Drone-based data collection is performed in two steps. The first step consists in simply locating survivors (e.g. the active devices at $n_{U}$ layer). Then, the second step consists in providing connectivity to devices. In this step, we should guarantee that a drone visits each $n_{U}$ layer nodes periodically in order to collect SOS data from survivor but also, potentially provide them with invigorating information. The droneassisted data exchange is more detailed in Section VI.

\section{FLY-COPE GROUND COMPONENT}

The ground component builds on heterogeneous devices that self-organize to leverage cooperative communications. This mechanism, called COPE [6] is rather simple. At bootstrap, every node activates its lowest communication interface $n_{1}$ and broadcast periodically some Hello messages in order to discover nearby nodes also active on this layer. Then, by exchanging neighbor information with them, every node is able to determine cliques, i.e. a set of nodes in which every node can directly communicate with every other one. For instance, on Figure 3, at $n_{1}$ layer, two cliques exist, the first one includes nodes $S_{1}, S_{2}, S_{3}$ and $S_{4}$ and the second one gathers nodes $S_{6}$, $S_{7}$ and $S_{5}$. 


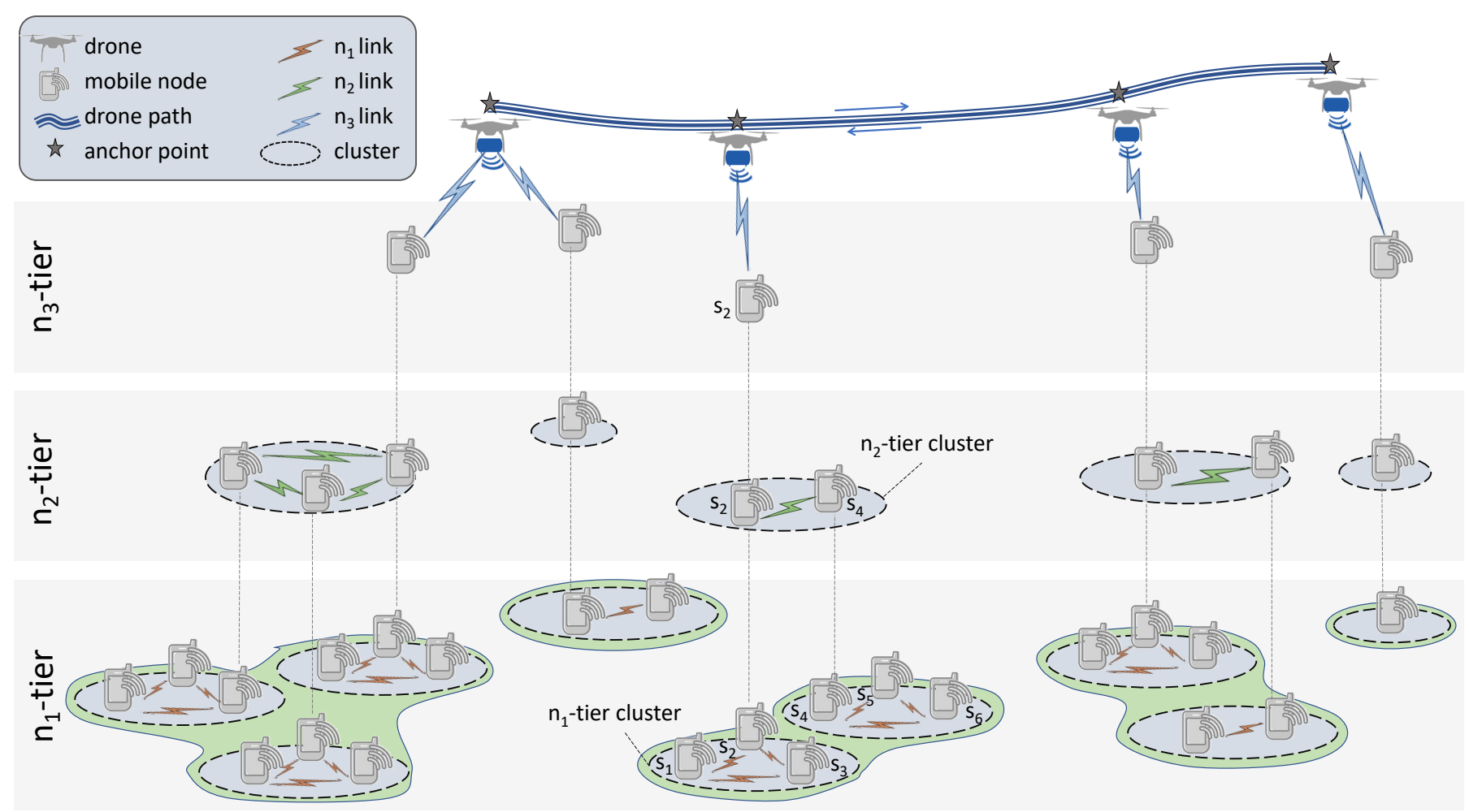

Fig. 1: Multi-tier network architecture: (example of three communication technologies)

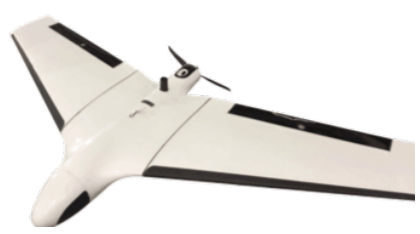

(a) Fixed Wing drone

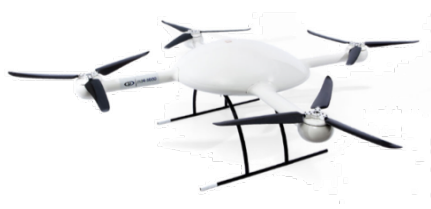

(b) Multi-rotor drone
Fig. 2: Different kinds of drones

Once cliques are identified at a layer $n_{i}$, nodes in each clique self-organize to determine when they will represent the $k_{i}$ nodes of $n_{i}$ clique nodes in $n_{i+1}$ layer and how long. To do so, each node will gather data for all nodes $j$ of the clique (identifier $j$ and energy budget $E_{j}$ of every node in the clique) and activate its $n_{i+1}$ communication interface once in periodic time slot of duration $\tau$. Nodes will take turn as a representative in the order defined by their identifier (the lowest id will take turn first) and for a duration $t_{j}$ proportional to its energy budget, e.g for $t_{j}=\tau \times \frac{E_{j}}{\sum_{k_{i}=0}^{N} E_{k_{i}}}$. The same process is applied at layer $n_{i+1}$ between all $n_{i}$ representative nodes, and so on, till the selection of $n_{U-1}$ representative nodes that will activate their $n_{U}$ communication interface and directly communicate with the drones. Figure 3 shows the duty cycle of nodes for the deployment illustrated at the top of the figure. In the clique composed by nodes $S_{1}, S_{2}, S_{3}$ and $S_{4}$, all nodes have a similar energy budget and thus they activate their $n_{2}$ communication interface for equal duration of time during each time slot. But in the second clique, node $S_{5}$ has twice more energy than nodes $S_{6}$ and $S_{7}$ and thus it is activated twice longer in each period.

Such a cooperative communication based self-organization scheme allows all nodes to stay alive as long as possible as Figure 4 shows as compared to a selfish approach in which each node just activates all its communication interface. The full evaluation results are available in [6].

\section{FLY-COPE AERIAL COMPONENT}

As detailed in Section IV, this section detailed the aerial component of FLY-COPE. We assume it is split in two phases: i) a search phase that aims to locate upmost layer nodes and identify the nodes they represent and ii) a communication phase. In both phases, drones are equipped with $n_{U}$ communication interface and activate it.

The search phase simply sends a drone that methodically spans all the damaged area to locate any survivor as shown by Figure 5. The fixed-wing drone follows an $S$-shaped route, whose curvature guarantees that all nodes can be discovered. At the end of this phase, the drone has eventually collected information from all $n_{U-1}$ layer representative nodes and can locate them and estimate the service time for each of them, the service time being proportional to the number of nodes represented.

The collected data allows the computing of the trajectory to be followed by the drone in the second phase. It is performed in two steps: I) compute anchor points positions and ii) compute travel salesman (TSP) trajectory that visit all these 

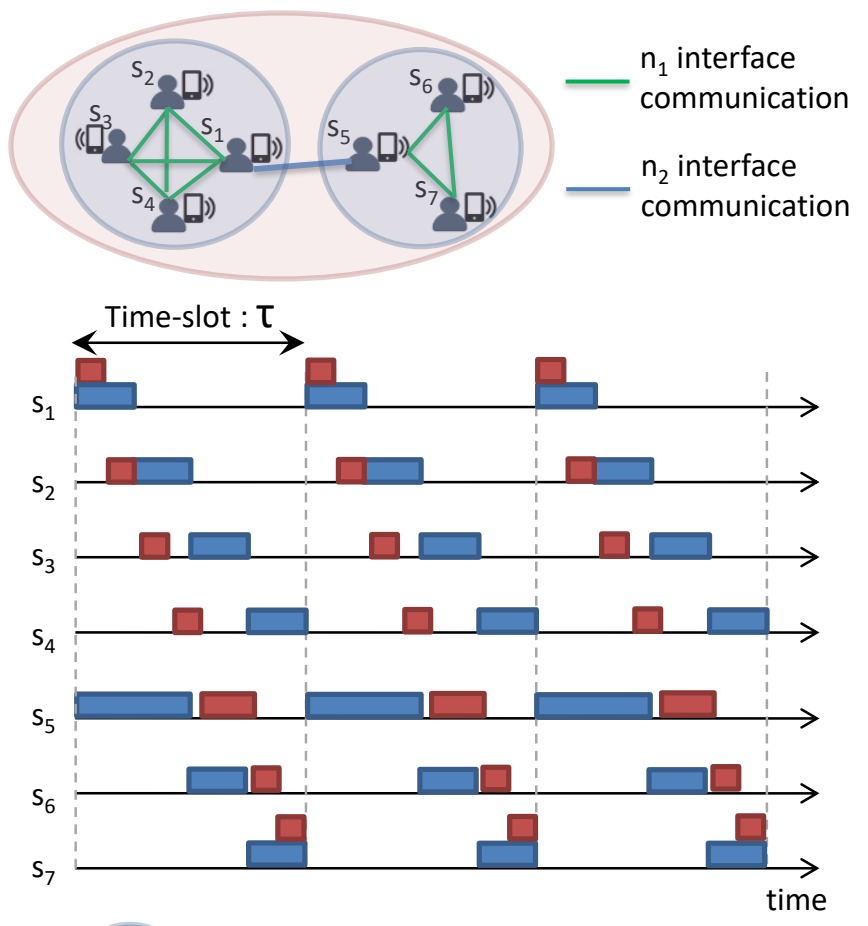

clique $\square n_{3}$ interface diffusion period

zone $\square \mathrm{n}_{2}$ interface diffusion period

Fig. 3: Example of self-organization and time sharing

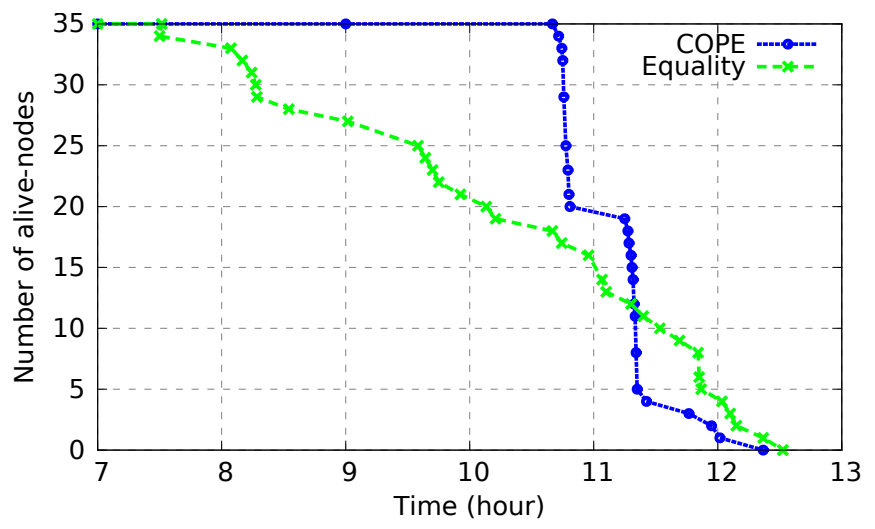

Fig. 4: Node lifetime

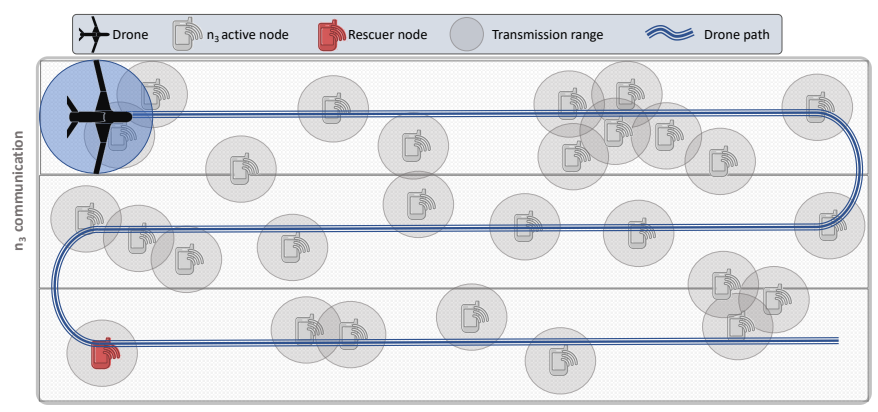

Fig. 5: Search Phase anchors points, also referred as a close-enough TSP (CE-TSP. Anchor points are positions where a drone should hover to collect data from ground nodes. It can be either at the vertical of each node discovered during the first phase or positions from which it can cover several ground nodes at once. For instance, on Figure 6a, stars represent the positions of anchor points. Consequently, given such anchor points as an input to a path planning algorithm, the shortest path that visits all these points is then constructed. The design of the path planning algorithm focuses especially on the energy consumption of the drone. That is, such algorithms aim at reducing the tour length of an UAV, hence the time it takes to fly over a disaster area and collect the data from the nodes. In fact, such an approach reduces the energy expenditure of the UAV. More specifically, leveraging the cooperative communication and data relay protocol among the nodes underlying the UAV yields to reduce the number of points a drone should visit. In fact, identifying anchor points from which a drone can serve more than one node results in a lower number of stops for the drone. The drone then follows such a path and collects data (Figure 6b).

Figure 7 shows an overview of the saving that are achieved in terms of path duration for different approaches. TSP approaches consider having the drone to visit every node while in CETSP (Close Enough TSP) approaches, only anchor points are visited.

COPE-FLY approaches consider that only representative nodes elected based on the COPE-FLY approach described in Section V are visited while other approaches visit all survivor nodes. Complete evaluation and comparison of these approaches are available in [12].

\section{OPEn CHALLENGES}

In this section, we browse a non exhaustive list of some open challenges and potential research directions to investigate to complete and/or improve our architecture efficiency.

\section{A. Ground component}

Survivor and rescuer mobility: We have assumed that survivors have a low mobility since they could be wounded and buried under rubble. Our scheme periodically re-computes cliques and time to serve as a representative at each communication layer and thus is assumed to be reliable to faster mobility schemes but this should be better investigated. In addition, as in our approach, all nodes are not all active at the same time, rescuers or drones can be in range of a survivor at a given time but not of its representative during this period and thus messages can be missed. From the first studies we have realized, the amount of these losses is low but it strongly depends on the survivors and drones/rescuers mobility and speed.

Multi-clique leveraging: Currently, our self-organization simply gathers nodes based on cliques naturally formed by communication links. Although clusters could be formed instead in which the connectivity between cluster members 


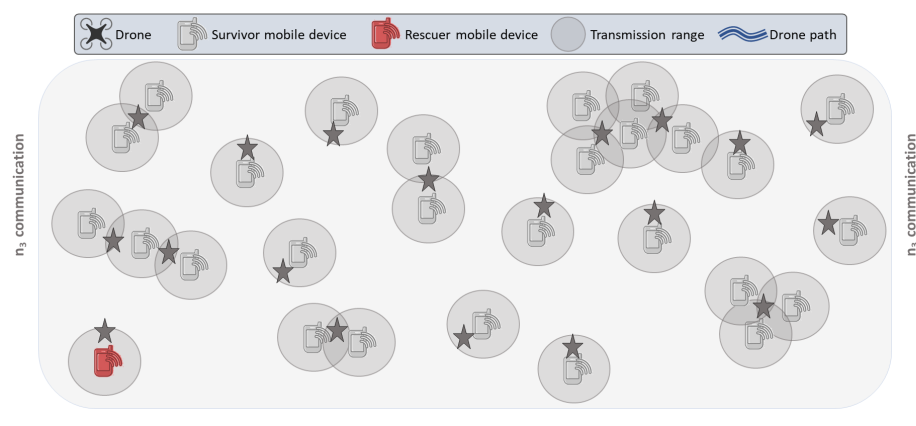

(a) Communication phase - anchor points

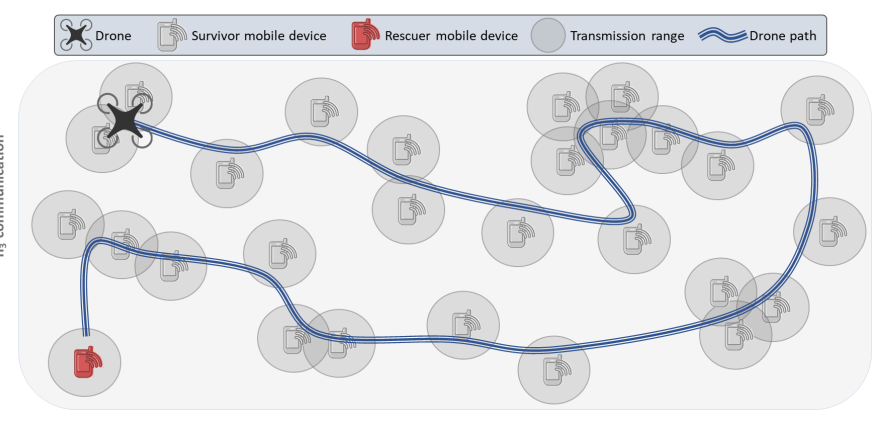

(b) Communication phase - Path

Fig. 6: COPE-FLY aerial phases

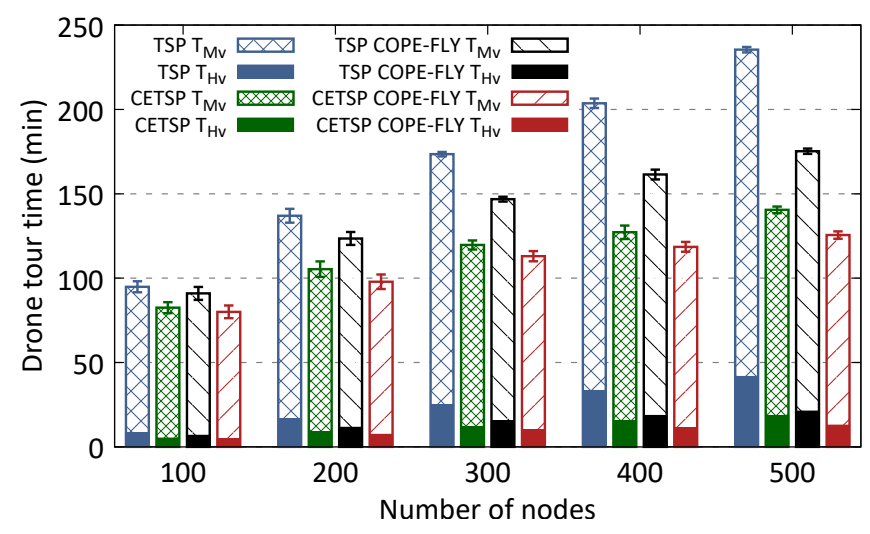

Fig. 7: Time spent by the drone for hovering $\left(T_{H T}\right)$ and for moving $\left(T_{M V}\right)$ with different approaches

does not represent a complete graph, we claim that such a solution would bring unnecessary complexity in the computing of the time spent as group representative for each node. However, it may happen that a node belongs to several cliques, as depicted by Figure 8 . Currently, we simply assume that such nodes will choose to belong only to the smallest clique for balance purpose but smarter schemes that dynamically adapt the clique membership could be investigated.

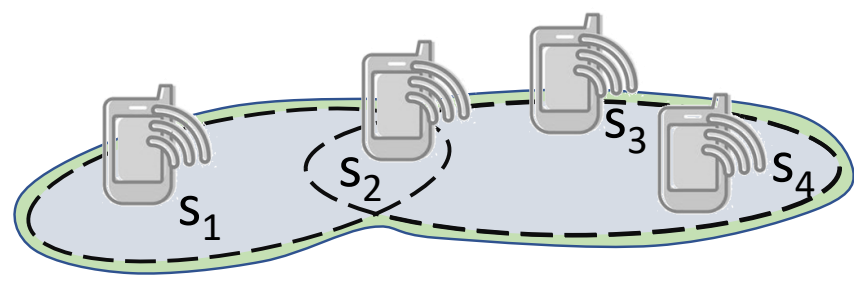

Fig. 8: Example of clique multi-membership

Devices heterogeneity: Our approach is robust to a set of devices featuring different amount of remaining energy. We experimentally verified our assumptions on the possibility to rank communication technologies based on their ranges and costs [24] for a homogeneous set of devices. Nevertheless, as it has been highlighted by a recent study [25], the signal reception quality of a given signal greatly depends of the hardware used and of different settings. Yet, our assumption may not be verified in all cases. But, as it only uses connectivity information between devices to form cliques and so is robust to imperfect propagation ranges and unilateral links. Not verifying this assumption would result in nonoptimal energy consumption. So, more investigation should be performed to quantify the impact of this heterogeneity in devices and propagation ranges. In addition, our goal is to rely opportunistically on all available devices that could support the data collection at the rescue center. Therefore, it could include other pieces of infrastructures such as base stations that are still active and powered but disconnected to the core network. They could still act as strong relays since benefiting from an infinite energy reserve. In our scheme, such strong access points will naturally be representative of a clique for all other nodes but our scheme does not leverage its potential longer range and more likely connectivity with several cliques.

Unavailability of some communication interfaces: In our approach, we have assumed that all devices are equipped with all same communication interfaces but for different reasons, this could not be the case. Some interface may be unavailable because the device has not been equipped with it, or it is damaged or the environment does not affect all interfaces similarly. This is thus worth integrating in our scheme the fact that all devices can represent the clique for a given layer.

\section{B. Aerial component}

Multi-drones: Our scheme currently investigates the use of a single drone and could be simply extended to the use of several drones by sharing between them the areas or anchor nodes to cover. However, due to the dynamics of the anchors at the upmost layers, such a static splitting might not be optimal and a dynamic area responsibility could be set as in [26]. 


\section{Dynamic 3D drones path planning:}

Currently, we assume that thanks to the upmost layer nodes discovery and location, drones are able to compute anchor points and the best traveling path visiting all these anchor points. We also assume that at each visit, nodes inform the drone about change in upmost layer representative allowing it to recompute its path. The path computing is realized in 2 steps: first compute the anchor points and then draw a (Close Enough) traveling salesman problem trajectory. This does not consider the drone autonomy nor a drastic change in representative positions and computes a $2 \mathrm{D}$ path. But drone coverage depends on the drone altitude and speed; the drone presents a highly flexible 3-D mobility and the higher the altitude, the larger the coverage but the higher the energy consumption [22]. And this could change the anchor points determination since by flying at a higher altitude, the drone will cover more nodes at a time but will consume more energy. An open problem is thus to determine the best energy-efficient and minimum-time 3D path to travel the area as fast as possible while still remaining in range of each survivor long enough to assure full servicing. This path should jointly investigate the drone trajectory and the location of the anchor nodes that could dynamically be adapted with drone altitude, while still integrating the pitstop duration at each anchor point, which has a mandatory minimum duration and should be proportional to the number of nodes to serve at this position [13].

\section{CONCLUSION}

This paper shows the potential of cooperative communications in post-disaster rescue operations. It introduces the FLYCOPE architecture which is a complete self-organized cooperative mechanism. FLY-COPE enables the spontaneous emergence of an efficient bilateral data communication between victims and rescuers. FLY-COPE is a global modular concept which unit components have been evaluated but it paves the ways to several exciting research directions, raising challenges of different kinds (optimization, networking, hardware, etc) that we plan to investigate in the future.

\section{ACKNOWLEDGEMENTS}

This work has been partially supported by the PIPA project and the CPER DATA.

\section{REFERENCES}

[1] S. F. Ochoa and R. Santos, "Human-centric wireless sensor networks to improve information availability during urban search and rescue activities," Information Fusion, vol. 22, pp. 71-84, 2015.

[2] A. Martín-Campillo et al., "Evaluating opportunistic networks in disaster scenarios," J. of Network and computer applications, 2013.

[3] L. E. Quispe and L. M. Galan, "Behavior of ad hoc routing protocols, analyzed for emergency and rescue scenarios, on a real urban area," Expert systems with applications, 2014.

[4] P. Yuan et al., "Recent progress in routing protocols of mobile opportunistic networks: A clear taxonomy, analysis and evaluation," JNCA, 2016.

[5] Z. Lu, G. Cao, and T. La Porta, "Teamphone: Networking smartphones for disaster recovery," IEEE Trans. on Mobile Computing, vol. 16, no. $12,2017$.
[6] F. Mezghani and N. Mitton, "Alternative opportunistic alert diffusion to support infrastructure failure during disasters," Sensors, 2017.

[7] "How drones aid in disaster response," 2019. [Online]. Available: https://www.precisionhawk.com/blog/how-drones-aid-in-disasterresponse

[8] "5 ways drones are being used for disaster relief." [Online]. Available: https://safetymanagement.eku.edu/blog/5-ways-drones-arebeing-used-for-disaster-relief/

[9] "Humanitarian in the sky: drones for disaster response." [Online]. Available: https://www.virgin.com/virgin-unite/businessinnovation/humanitarian-sky-drones-disaster-response

[10] J. Lyu, Y. Zeng, R. Zhang, and T. J. Lim, "Placement optimization of UAV-mounted mobile base stations," IEEE Communications Letters, no. 3, pp. 604-607, 2017.

[11] J. Lyu, Y. Zeng, and R. Zhang, "Cyclical multiple access in UAV-aided communications: A throughput-delay tradeoff," IEEE Wireless Comm. Letters, vol. 5, no. 6, pp. 600-603, 2016.

[12] F. Mezghani, P. Kortoçi, N. Mitton, and M. Di Francesco, "A Multitier Communication Schemefor Drone-assisted Disaster Recovery Scenarios," in PIMRC 2019 - IEEE International Symposium on Personal, Indoor and Mobile Radio Communications, Istanbul, Turkey, Sep. 2019.

[13] F. Mezghani and N. Mitton, "Open problem: Energy-and time-efficient dynamic drone path planning for post-disaster network servicing," in ODS 2018 - International Conference on Optimization and Decision Science, Taormina, Italy, Sep. 2018.

[14] F. Wex, G. Schryen, S. Feuerriegel, and D. Neumann, "Emergency response in natural disaster management: Allocation and scheduling of rescue units," European Journal of Operational Research, 2014.

[15] N. Chakchouk, "A survey on opportunistic routing in wireless communication networks," IEEE Communications Surveys \& Tutorials, 2015.

[16] H. Nishiyama et al., "Relay-by-smartphone: realizing multihop deviceto-device communications," IEEE Comm. Mag., 2014.

[17] S. D. Ramchurn, F. Wu, W. Jiang, J. E. Fischer, S. Reece, S. Roberts, T. Rodden, C. Greenhalgh, and N. R. Jennings, "Human-agent collaboration for disaster response," Autonomous Agents and Multi-Agent Systems, 2016.

[18] L. Gupta et al., "Survey of important issues in UAV communication networks," IEEE Comm. Surveys \& Tutorials, 2016.

[19] Y. Zeng, R. Zhang, and T. J. Lim, "Wireless communications with unmanned aerial vehicles: opportunities and challenges," IEEE Com. Mag., 2016.

[20] L. Reynaud and I. Guerin Lassous, "Improving the performance of challenged networks with controlled mobility," Mobile Networks and Applications (ACM/Springer), Special issue Ad Hoc Networking and Emerging Applications, January 2017.

[21] M. Mozaffari, W. Saad, M. Bennis, and M. Debbah, "Unmanned aerial vehicle with underlaid device-to-device communications: Performance and tradeoffs," IEEE Transactions on Wireless Communications, 2016.

[22] D. Zorbas, L. D. P. Pugliese, T. Razafindralambo, and F. Guerriero, "Optimal drone placement and cost-efficient target coverage," Journal of Network and Computer Applications, 2016.

[23] M. Mozaffari, W. Saad, M. Bennis, and M. Debbah, "Efficient deployment of multiple unmanned aerial vehicles for optimal wireless coverage," IEEE Communications Letters, 2016.

[24] F. Mezghani and N. Mitton, "Benchmarking smartphone performances for cooperative disaster alert diffusion," in Adhoc Now, 2018.

[25] C. Bertier, M. Dias De Amorim, F. Benbadis, and V. Conan, "Modeling Realistic Bit Rates of D2D Communications between Android Devices," in ACM International Conference on Modeling, Analysis and Simulation of Wireless and Mobile Systems (MSWiM), Miami, United States, Nov. 2019.

[26] T. Razafindralambo, N. Mitton, A. Carneiro Viana, M. Dias De Amorim, and K. Obraczka, "Adaptive Deployment for Pervasive Data Gathering in Connectivity-Challenged Environments," in Eighth Annual IEEE International Conference on Pervasive Computing and Communications (PERCOM). Mannheim, Germany: IEEE, Mar. 2010, pp. 51-59. 